Supporting Information

\title{
A Quantitative Assay of Sodium Triacetoxyborohydride
}

\author{
Michael J. Zacuto, * Joseph Perona, and Robert Dunn
}

Drug Substance Development, Celgene Corporation, 556 Morris Avenue, Summit, NJ 07901, USA

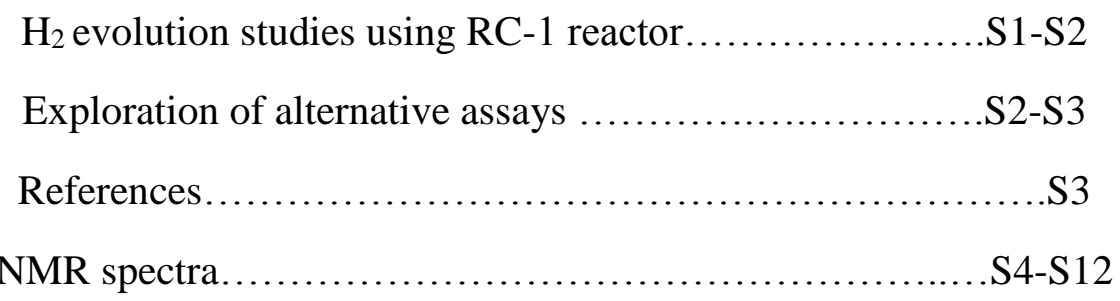

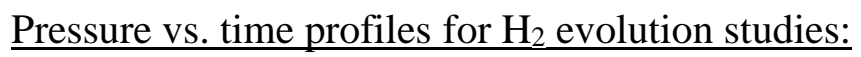

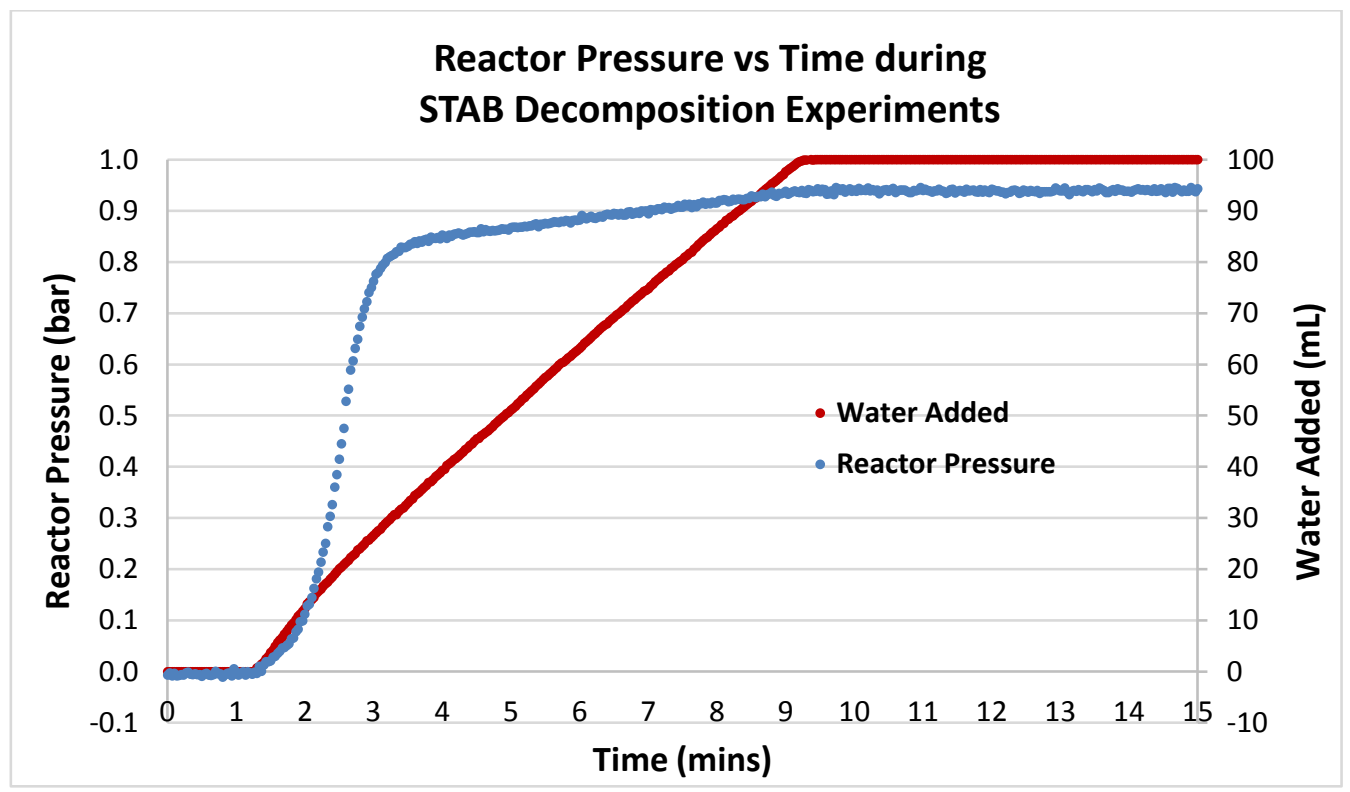

Figure: Typical plot of reactor pressure vs time during the $\mathrm{H}_{2}$ evolution experiments. 


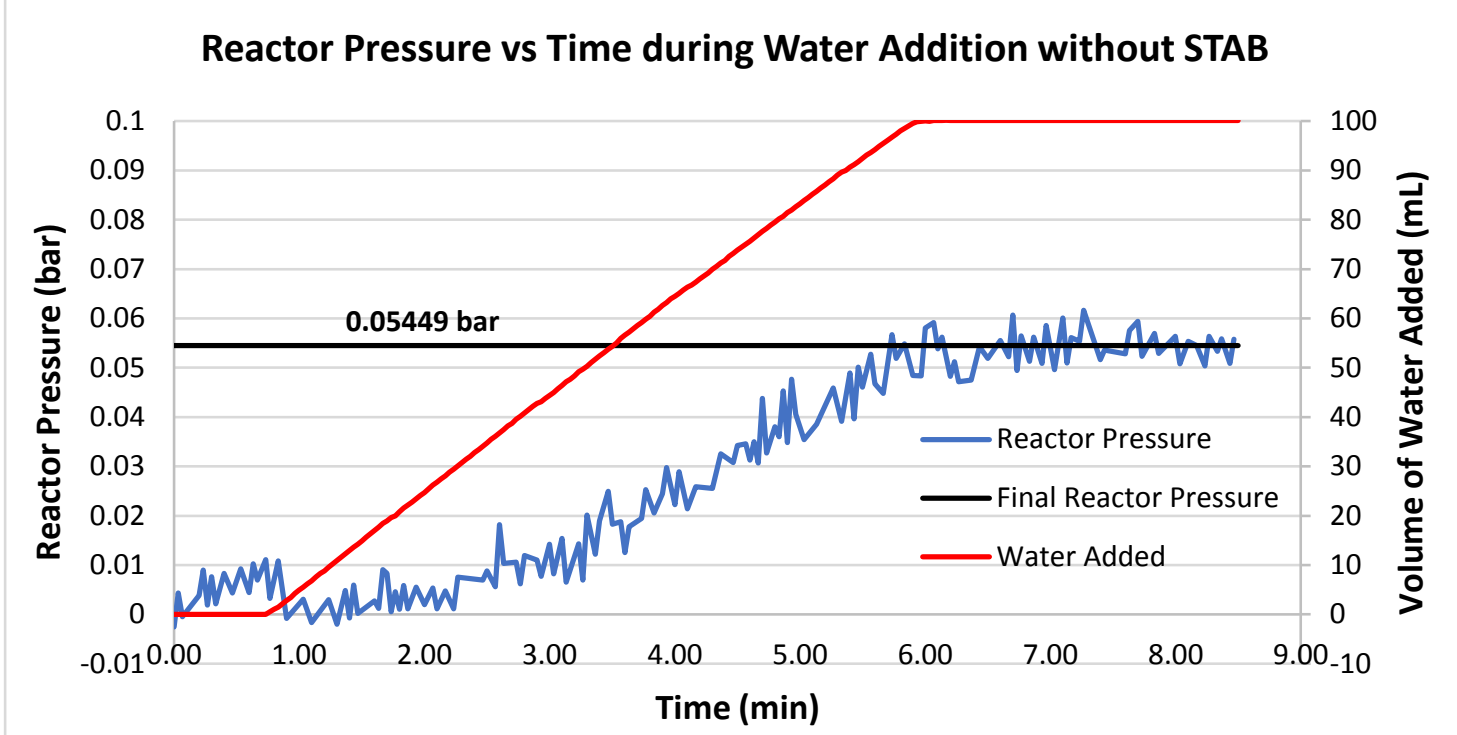

Figure: Plot of reactor pressure vs time during a water addition with no STAB present in the system. This was done to determine the pressure increase due to the water addition into a closed system in our experiments. The pressure increase from the water addition was found to be 0.05449 bar.

Alternative assays examined:

Elemental analysis $(\mathrm{C}, \mathrm{H}, \mathrm{N}, \mathrm{B})$ data was collected for various samples of STAB, but we were unable to correlate it reliably to our assay data. Although we did not study this extensively, it is possible that the hygroscopicity of STAB contributed to error in the measurements.

Quantitative ${ }^{1} \mathrm{H}$ NMR techniques were explored, whereby a mixture of STAB and an internal standard (1,3,5- trimethoxybenzene) was dissolved and a spectrum was acquired. We found that in a variety of solvents $\left(d_{7}-\mathrm{DMF}, d_{8}\right.$ - THF, and $\left.\mathrm{CD}_{3} \mathrm{CN}\right)$, acetate methyl groups associated with STAB partially overlapped with those from acetic acid, as well other unidentified (but presumably related) singlets. This overlap complicated accurate quantification via this 
technique. Furthermore, accurate detection and integration of to the B-H moiety was difficult due to complex splitting patterns due to B-H coupling and boron isotopes. ${ }^{1}$

Furthermore, STAB- mediated reduction of two iminium- based dyes (malachite green oxalate salt and Gentian violet) was attempted. Disappearance of color has been reported in the case of $\mathrm{NaBH}_{4},{ }^{2}$ akin to an indicator-based titration, due to the reduction of the imine. We confirmed these findings, and attempted to apply them to $\mathrm{NaBH}(\mathrm{OAc})_{3}$. The reaction with STAB (as judged by the disappearance of color), however, was slow and incomplete- requiring a large excess relative to the iminium species and therefore was unsuitable as an assay. A related approach was the reaction with $\mathrm{I}_{2}$. We found that at least two equivalents of STAB relative to $\mathrm{I}_{2}$ were required, ${ }^{3}$ and the color disappearance was gradual, rendering this approach unsuitable as an indicating reaction.

\section{References:}

\footnotetext{
${ }^{1}$ Šljukić, B.; Santos, D. M. F.; Sequeira, C. A. C.; Bank, C. E. Analytical monitoring of sodium borohydride. Anal. Methods, 2013, 5, 829.

${ }^{2}$ Bunton, C. A.; Huang, S. K.; Paik, C. H. Reactions of Triarylmethyl Carbocations with Hydride Donors and Other Nucleophiles. Tetrahedron Lett. 1976, 17, 1445.

${ }^{3}$ The evolution of gas (presumably $\mathrm{H}_{2}$ ) suggested that $\mathrm{STAB}$ reacted with both $\mathrm{I}_{2}$ and the liberated $\mathrm{HI}$.
} 


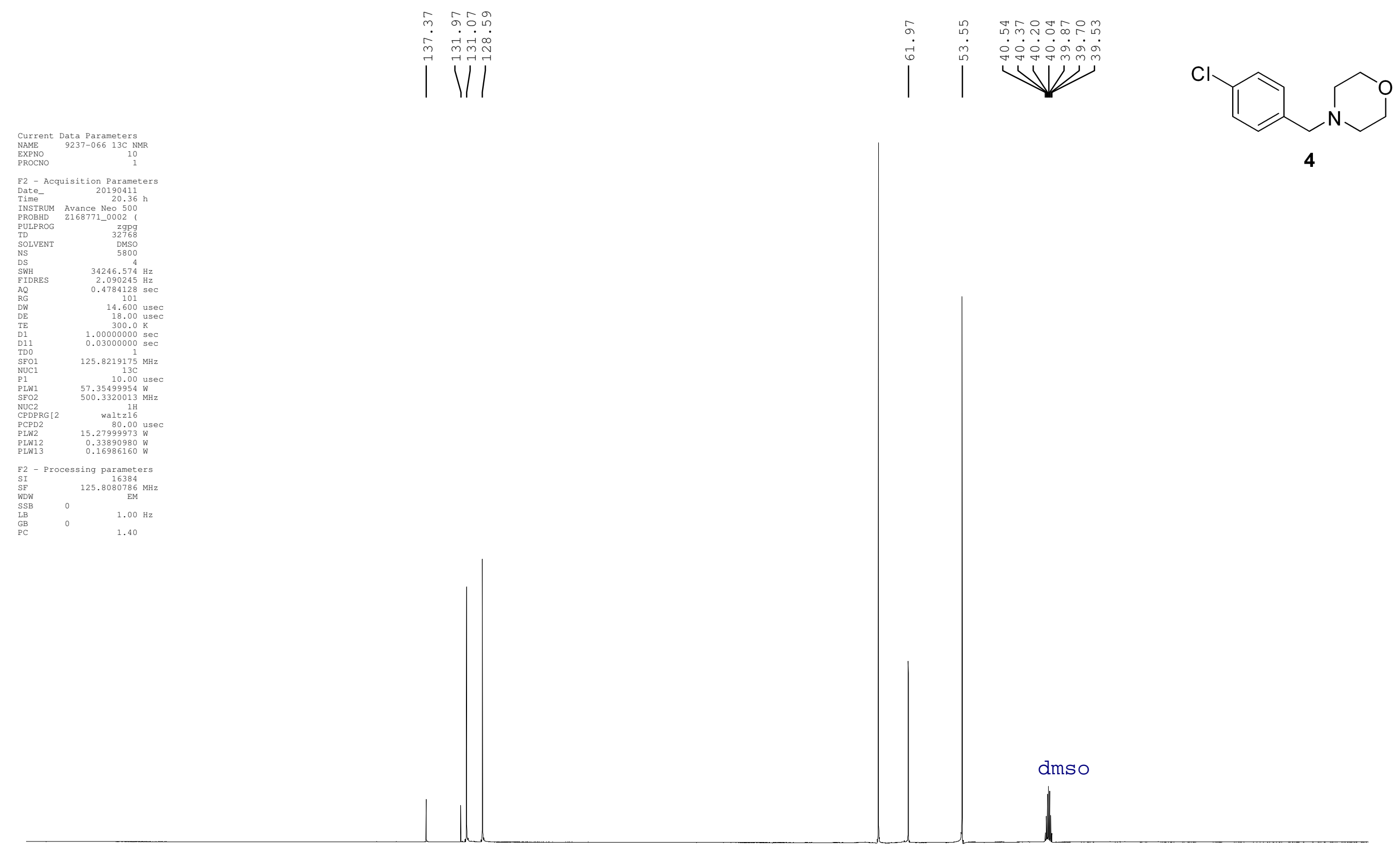




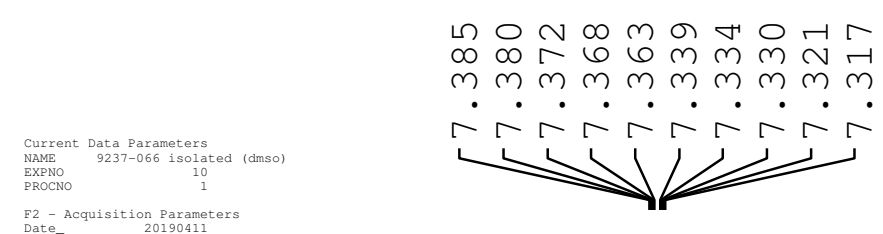

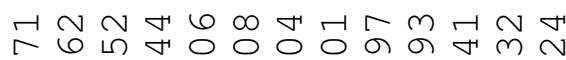
๓๓ $\dot{m} \dot{m} \dot{m} \dot{m} \dot{N} \dot{N} \dot{N} \dot{N} \dot{N} \dot{N}$ 将/।
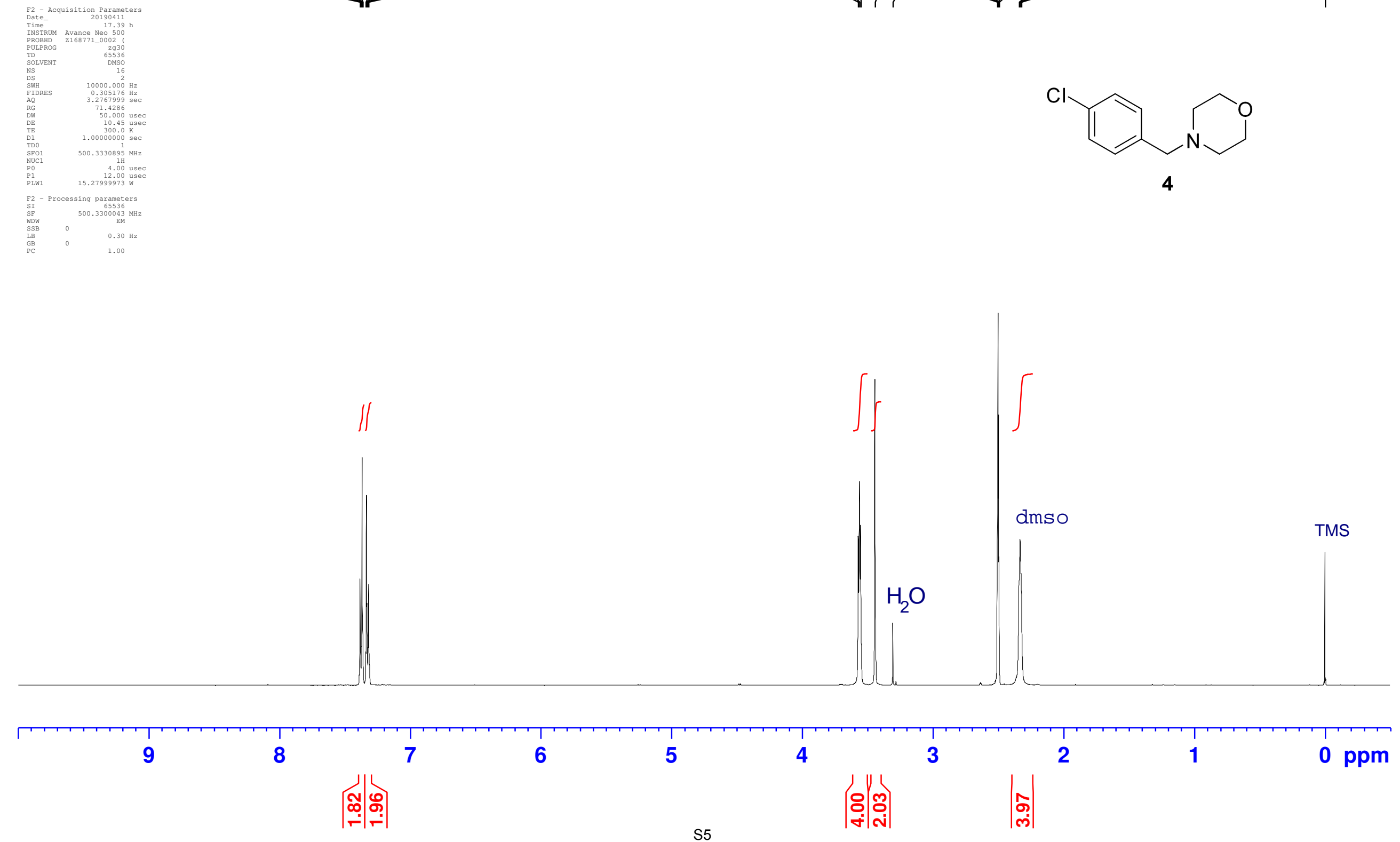


\section{$\mathrm{NaBH}(\mathrm{OAc})_{3}$ in $d_{T} \mathrm{DMF}$}

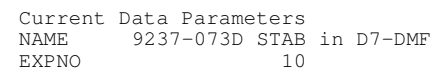

NAME
EXPNO
PROCNO

F2 - Acquisition Parameters
Date_ 20190122

Time
INSTRUM

INSTRUM
PROBHD
PUTPRO

PULPROG

SOLVENT
NS

DS
SWH
FTDRES

$\begin{array}{lr}6009.615 \mathrm{~Hz} \\ \text { FIDRES } & 0.091699 \mathrm{~Hz} \\ \text { AQ } & 5.4525952 \mathrm{sec}\end{array}$

$\begin{array}{lr}\text { AQ } & 5.4525952 \mathrm{sec} \\ \text { RG } & 144\end{array}$

$\begin{array}{lr}\text { DW } & 83.200 \text { usec } \\ \text { DE } & 6.50 \text { usec } \\ \text { TE } & 295.6 \mathrm{~K} \\ \text { D1 } & 1.00000000 \text { sec }\end{array}$

TDO

$=======$ CHANNEL $\mathrm{f} 1 \mathrm{1}=======$
SFO1
NUC1
P1

$\begin{array}{lr}\text { P1 } & 12.35 \text { usec } \\ \text { PLW1 } & 20.00000000 \text { W }\end{array}$

F2 - Processing parameters

$\begin{array}{lc}\text { SI } & 6556 \\ \text { SE } & 300.140000 \mathrm{MHz} \\ \text { WDW } & \mathrm{EM}\end{array}$

$\begin{array}{lll}\text { SSB } & 0 & 0.30 \mathrm{~Hz} \\ \text { LB } & 0 & \end{array}$

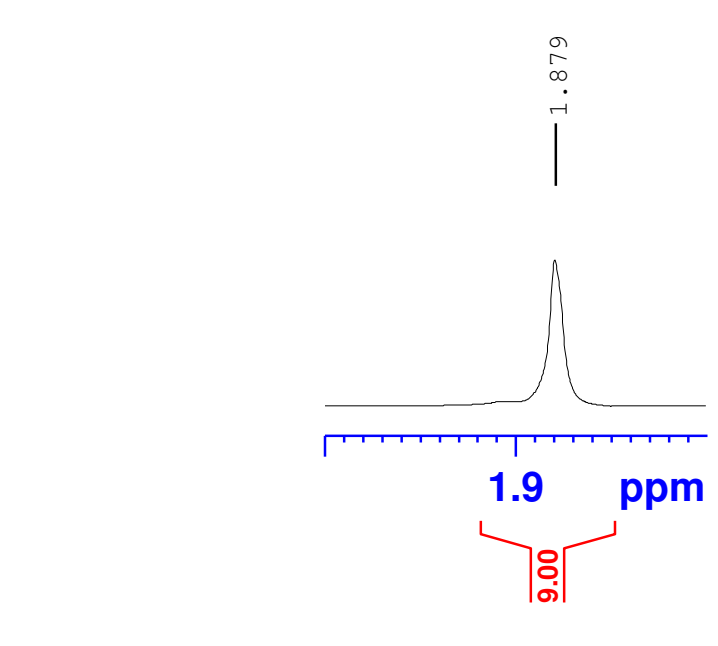

PC 1.00

1.00

DMF

DMF

$\downarrow \vee$

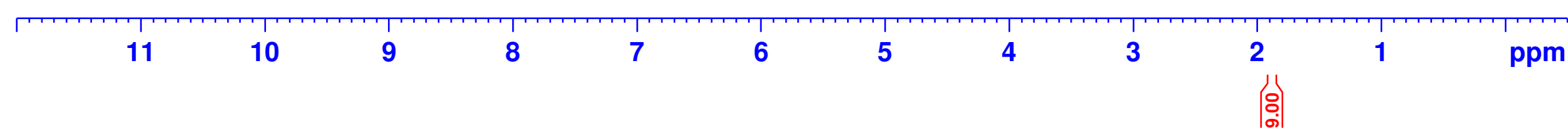


Current Data Parameters
NAME
EXPNO
9237-073D ACOH in d7-DMF

EXPNO
PROCNO

F2 - Acquisition Parameters
Date_ 20190122

$\begin{array}{lr}\text { Time } & 17.52 \\ \text { INSTRUM } & \text { spect }\end{array}$

$\begin{array}{lr}\begin{array}{l}\text { INSTRUM } \\ \text { PROBHD } \\ \text { PUIPROG }\end{array} & 5 \mathrm{~mm} \text { PABBO } \mathrm{\text {BB}} \\ \mathrm{zg}- \\ \mathrm{TD}\end{array}$

TD
SOLVENT

$\begin{array}{lc}\text { NS } & 16 \\ \text { DS } & 2 \\ \text { SWH } & 6009.615 \mathrm{~Hz} \\ \text { FIDRES } & 0.091699 \mathrm{~Hz} \\ \text { Re } & 5.4525952 \mathrm{~s}\end{array}$

$\begin{array}{ll}\text { FIDRES } & 0.091699 \mathrm{~Hz} \\ \text { AQ } & 5.4525952 \mathrm{sec} \\ \text { RG } & \end{array}$

$\begin{array}{lr}\text { AQ } & 5.4525952 \mathrm{sec} \\ \text { RG } & 144 \text { usec } \\ \text { DW } & 83.200 \text { usec } \\ \text { DE } & 6.50 \text { usec }\end{array}$

$\begin{array}{lr}\text { DE } & 6.50 \text { usec } \\ \text { TE } & 295.7 \mathrm{~K} \\ \text { D1 } & 1.00000000 \mathrm{sec} \\ \text { TD0 } & \end{array}$

$\begin{array}{ll}======= & \text { CHANNEL } \mathrm{f1}======= \\ \text { SFO1 } & 300.1418535 \mathrm{MHz}\end{array}$

$\begin{array}{lr}\text { NUC1 } & 1 \mathrm{H} \\ \text { P1 } & 12.35 \text { usec } \\ \text { PLW1 } & 20.00000000 \text { W }\end{array}$

F2 - Processing parameters

$\begin{array}{lc}\text { SI } & 65536 \\ \text { SF } & 300.140000 \mathrm{MHz} \\ \text { WDW } & \mathrm{EM}\end{array}$

$\begin{array}{llc}\text { WDW } & & \text { EM } \\ \text { SSB } & 0 & 0.30 \mathrm{~Hz} \\ \text { LB } & 0\end{array}$

${ }^{\mathrm{GB}}{ }^{\mathrm{PC}} 1.00$

$\int$

\section{$\mathrm{AcOH}$ in $d_{7}$-DMF}

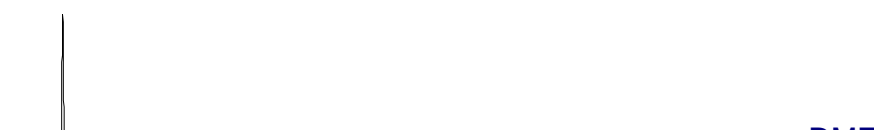

DMF

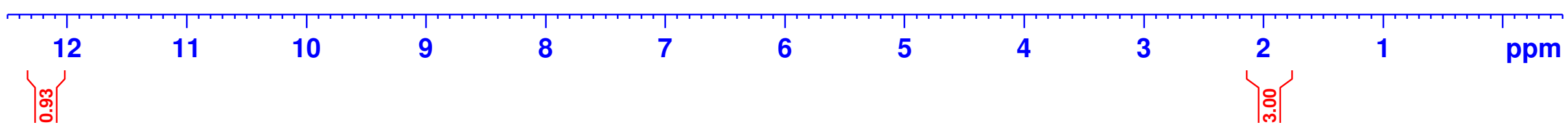



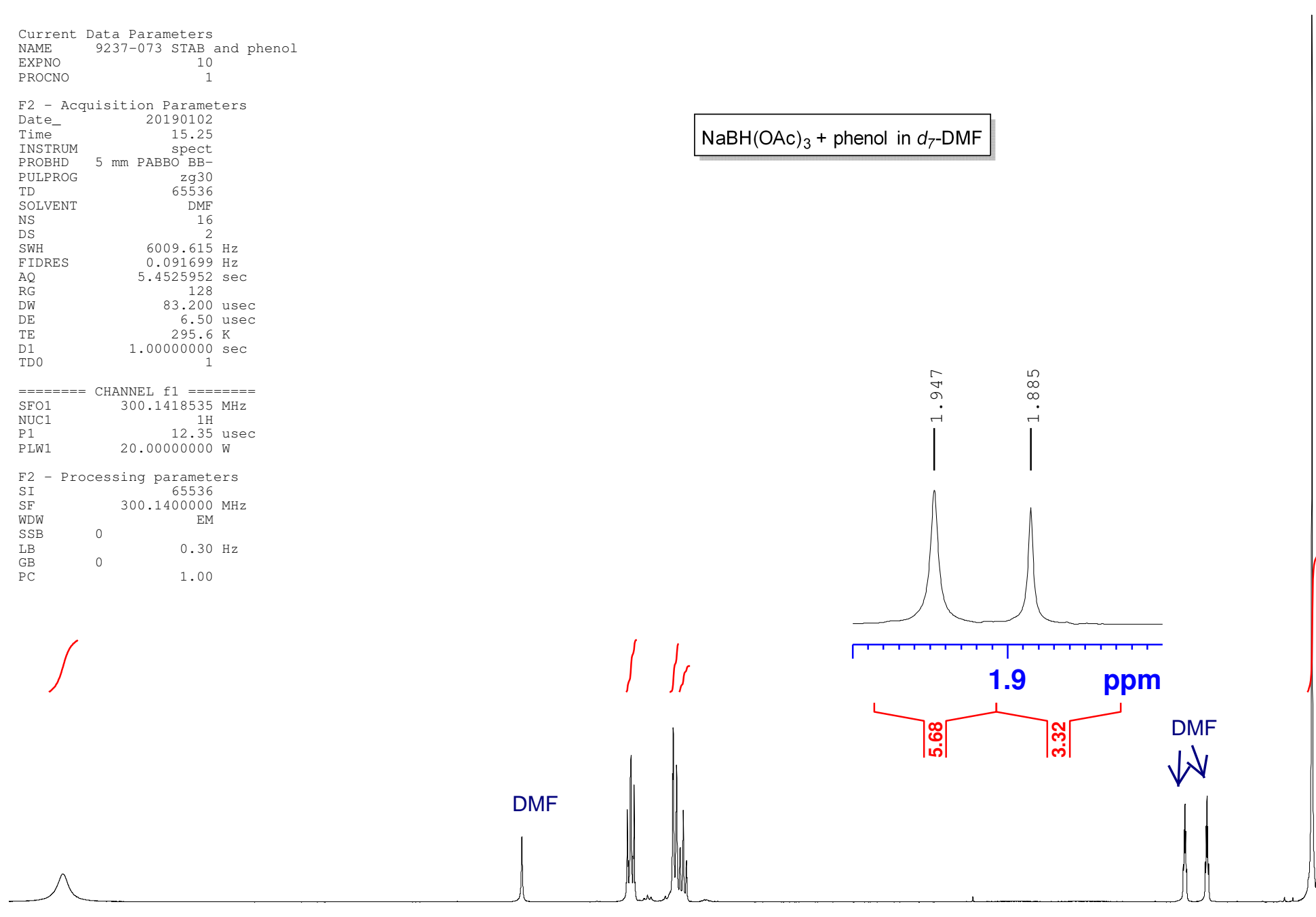

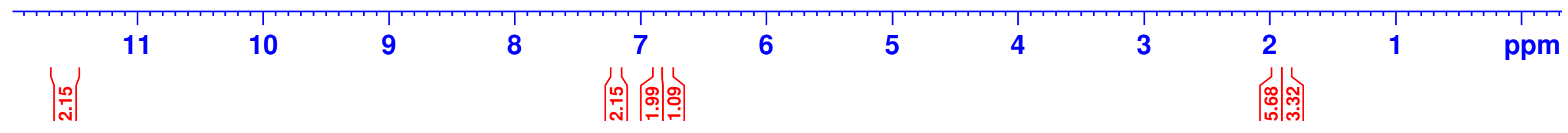




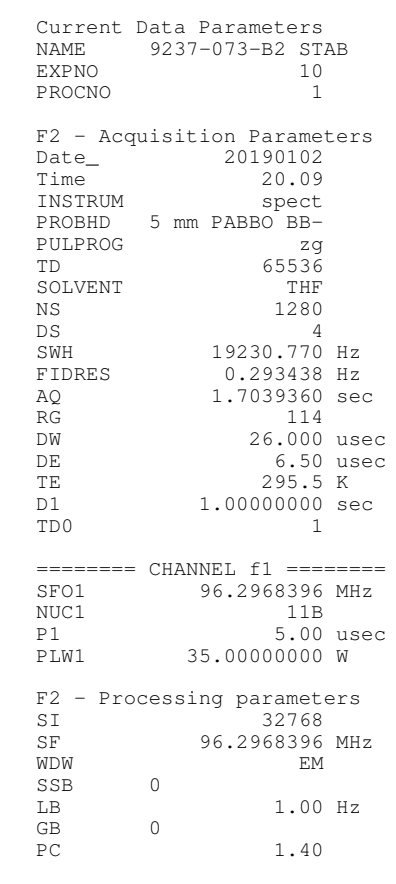

${ }^{11} \mathrm{~B} N M R$ of $\mathrm{NaBH}(\mathrm{OAc})_{3}$ in $d_{8}-\mathrm{THF}$

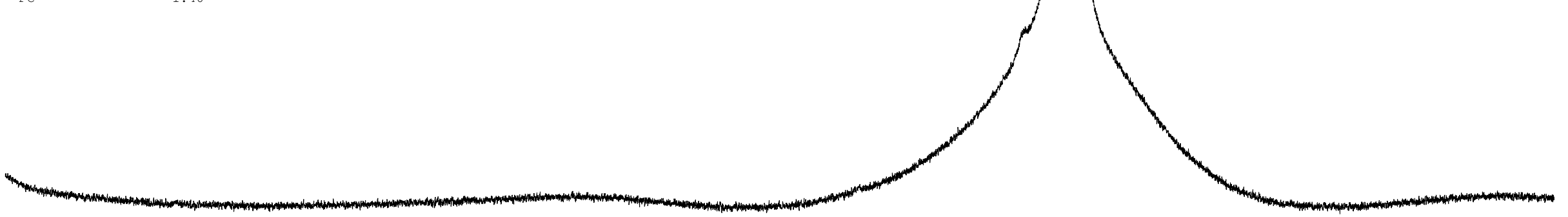

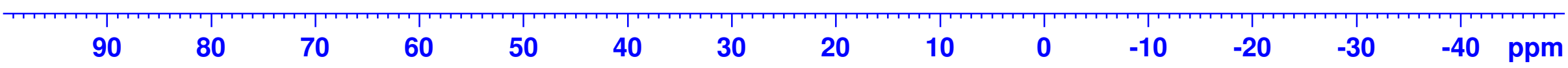




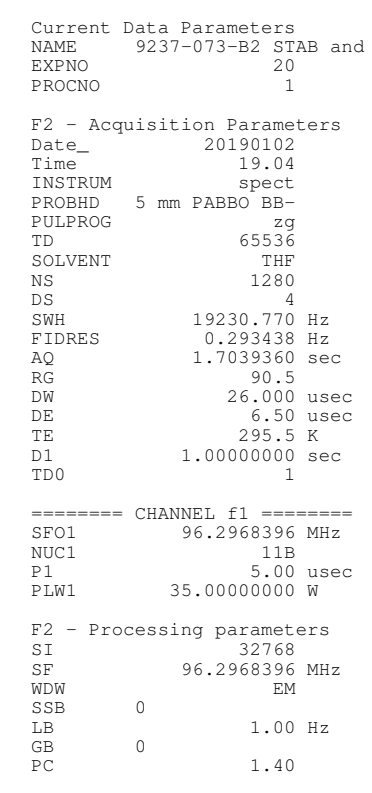

${ }^{11} \mathrm{~B} N M R$ of $\mathrm{NaBH}(\mathrm{OAc})_{3}+$ phenol in $d_{8}$ THF

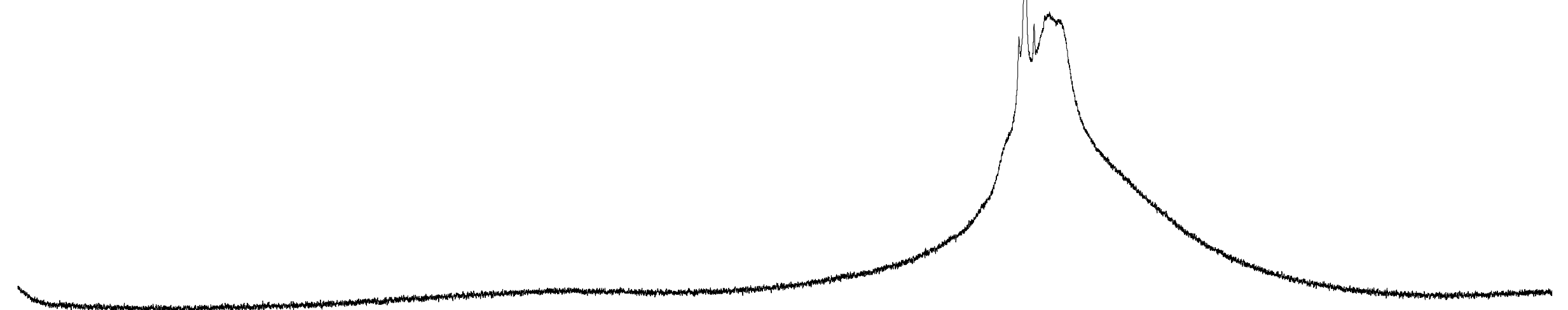




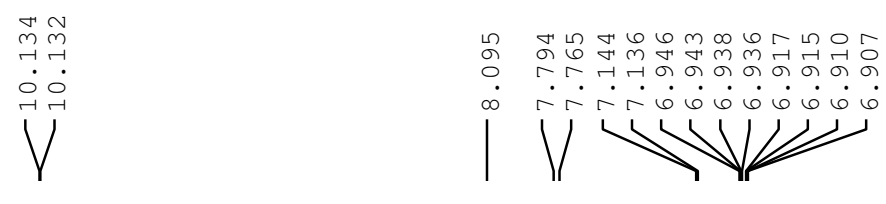

1H NMR of aldehyde 5 (CD3CN)
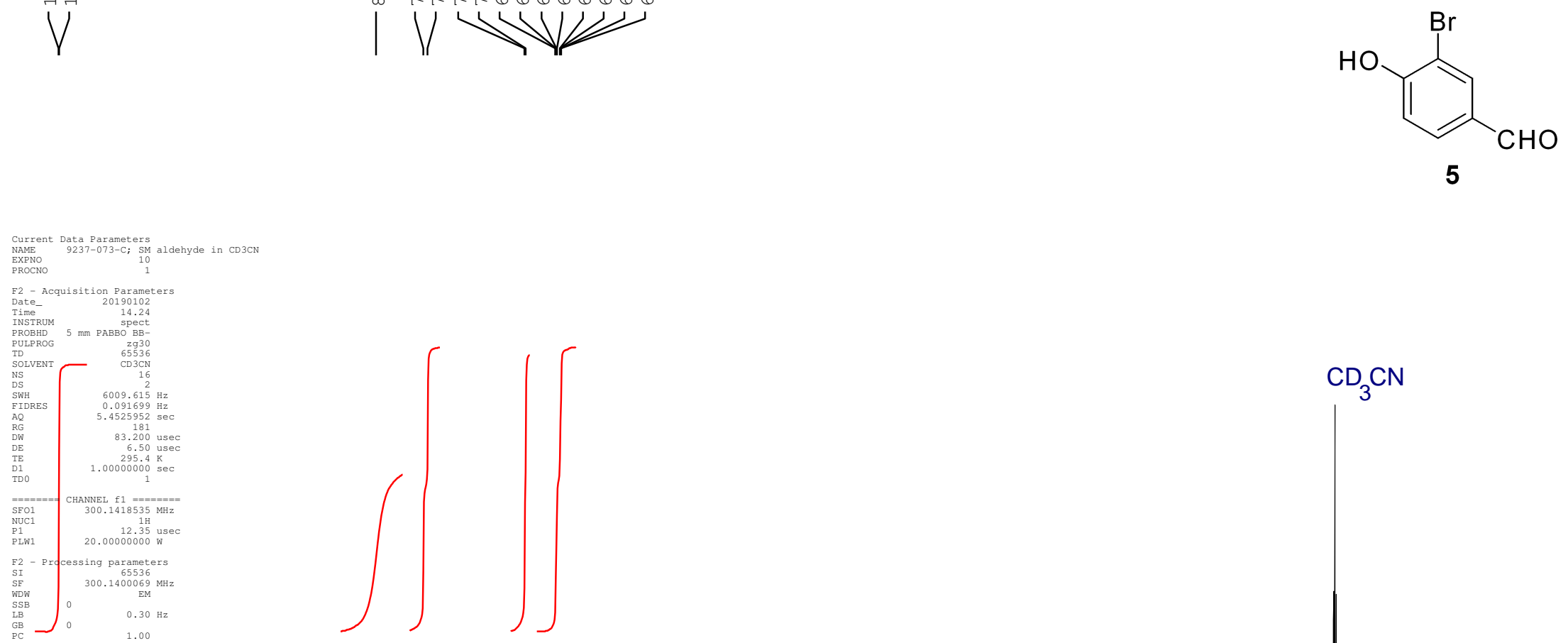

$\mathrm{CD}_{3} \mathrm{CN}$
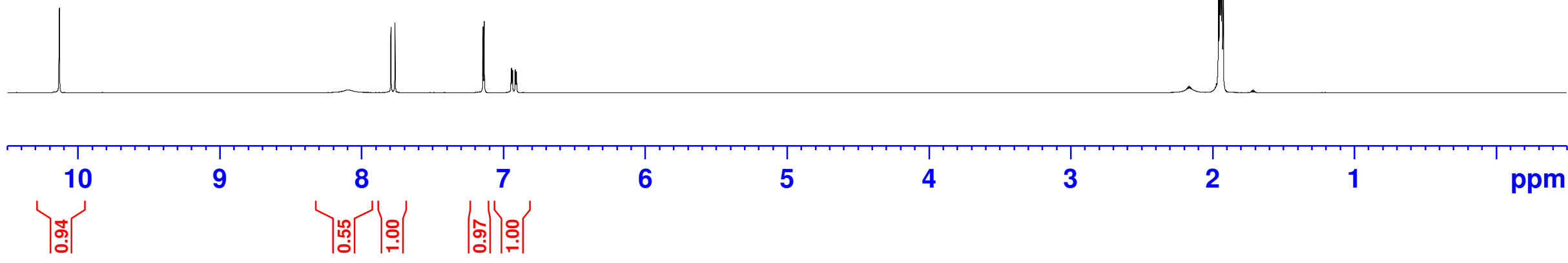

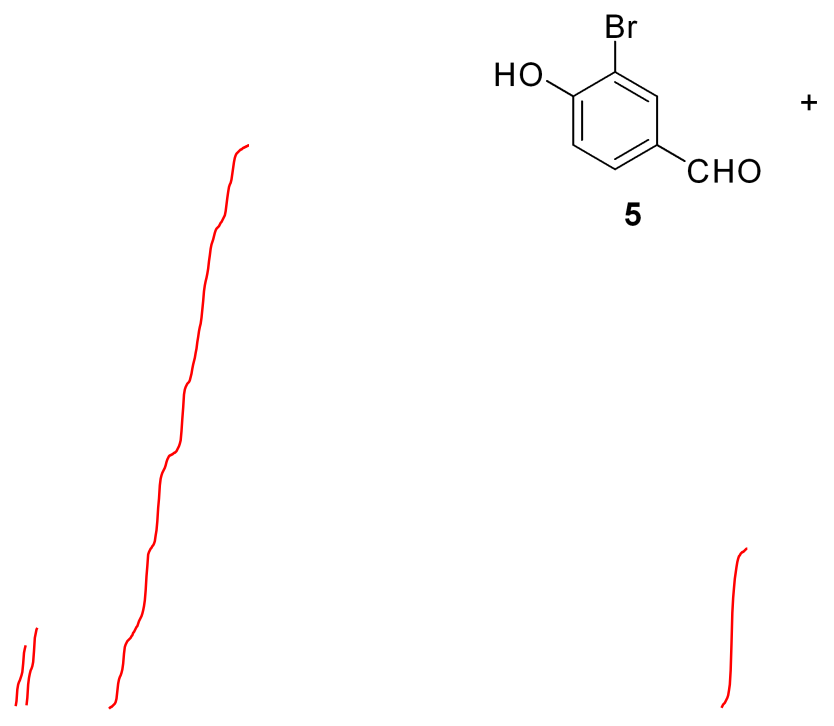

STAB

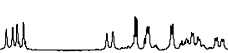

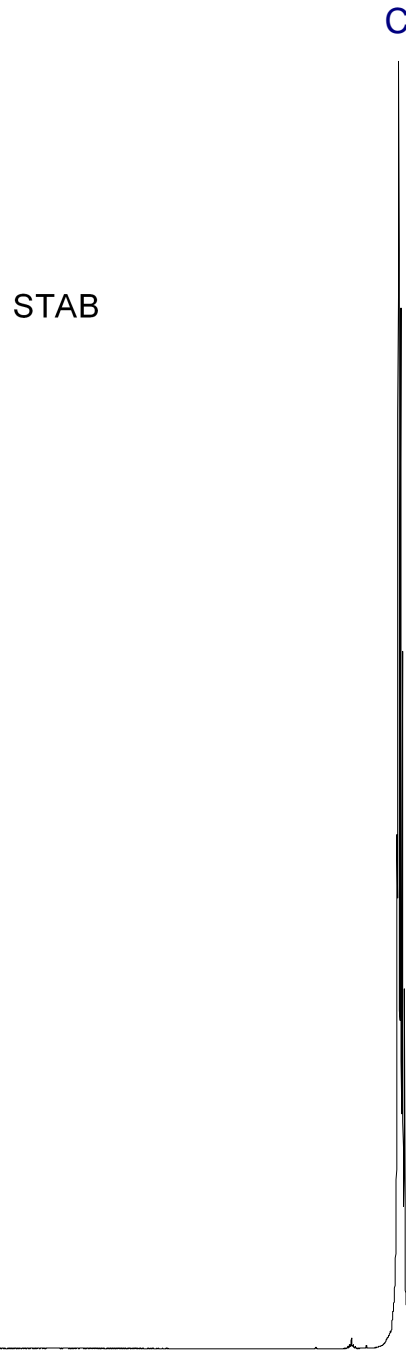

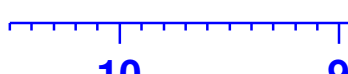

10

各各|
8

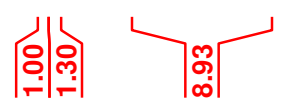

5

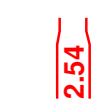

3

4

2

1

ppm 